\title{
NOMENCLATURE PROPOSÉE POUR LES ESPÈCES DÉCRITES DANS LA SOUS-FAMILLE DES OSTERTAGIINAE LOPEZ-NEYRA, 1947
}

\author{
M.-C. DURETTE-DESSET
}

RÉSUMÉ. Les espèces de la sous-famille des Ostertagiinae sont reclassées selon les critères suivants : type de bourse caudale (2-1-2 ou 2-2-1 ; longueur relative des côtes les unes par rapport aux autres) et synlophe.

Six genres sont reconnus : Marshallagia Orloff, 1933, Camelostrongylus Orloff, 1933, Ostertagia Ransom, 1907, Longistrongylus Le Roux, 1931, Spiculopteragia Orloff, 1933 et Teladorsagia Andreeva et Satubaldin, 1954.

Un index des genres et des espèces est proposé.

Mots-clés : Nomenclature. Nematoda. Ostertagiinae.

\section{Nomenclature proposed for the species described in subfamily Ortertagiinae Lopez-Neyra, 1947.}

SUMMARY. The species of the subfamily Ostertagiinae are reclassified according to the following characters: bursal ray pattern (2-1-2 or 2-2-1), relative length of ribs, and synlophe. Six genera are recognized: Marshallagia Orloff, 1933, Camelostrongylus Orloff, 1933, Ostertagia Ransom, 1907, Longistrongylus Le Roux, 1931, Spiculopteragia Orloff, 1933, and Teladorsagia Andreeva et Satubaldin, 1954. This paper does not intend to confirm or not the synonymies proposed by the authors. The paper also does not deal with problems of polymorphous species. Homonymies are indicated in chronological order and without creation of new names.

Indexes of genera and species are also produced.

Key-words: Nomenclature. Nematoda. Ostertagiinae.

\section{Introduction}

Parmi les Nématodes parasites de Vertébrés, les Ostertagiinae constituent un des cas les plus remarquables de confusion systématique. Les grandes sources d'erreur proviennent principalement de la description comme espèces de spécimens tératologiques (voir Drozdz, 1965), et de l'utilisation de taxons qui ne correspondent pas à la description originale, du fait que la bourse caudale n'est pas correctement étalée et que le synlophe n'est pas décrit.

Laboratoire de Zoologie-Vers associé au CNRS, Muséum National d'Histoire Naturelle, 61, rue Buffon, F 75231 Paris Cedex 05.

Accepté le 10 avril 1989. 
Cette note ne cherche pas à infirmer ou confirmer les synonymies données. Elle ne traite pas davantage des problèmes posés par les espèces polymorphes. Les homonymies sont indiquées dans l'ordre chronologique et sans création de nom nouveau.

Nous tentons, d'après les descriptions originales, de classer les espèces dans les genres que nous reconnaissons dans cette sous-famille, d'une part selon les critères que nous avons proposés (cf. Durette-Desset, 1982 et 1983), qui s'appuyaient principalement sur la disposition des côtes bursales, d'autre part sur la connaissance du synlophe.

Nous avons eu en effet l'opportunité d'observer plus récemment les synlophes des espèces-types des quatre genres Teladorsagia, Marshallagia. Camelostrongylus et Ostertagia. Le synlophe de l'espèce-type de Longistrongylus a été publié par Gibbons, 1977 ; celui de Spiculopteragia a été étudié par Ferté, 1987.

Il est donc devenu possible d'utiliser ce caractère pour la classification et, en particulier, de revalider le genre Camelostrongylus que nous pensions synonyme de Marshallagia par ses caractères bursaux, alors que son synlophe est de type Ostertagia.

Dans chaque genre, la liste des espèces est donnée par ordre alphabétique. Pour chaque espèce, nous indiquons quels sont les taxons qui ont été formés, puis les synonymies qui ont été proposées.

MARSHALLAGIA Orloff, 1933 (subgen.)

Espèce type: Ostertagia marshalli Ransom, 1207 (dés. de Travassos, 1937)

Elevé au rang générique par Travassos, 1937

\section{Synonymes:}

\section{Grosspiculagia Orloff, 1933 (subgen .)}

Espèce type : Ostertagia occidentalis Ransom, 1907 (dés. de Travassos, 1937). Elevé au rang générique par Sarwar, 1956. Synonymie générique: Durette-Desset, 1982

Ostertagiella Andreeva, 1957a.

Espèce type : Ostertagia occidentalis Ransom, 1907 (dés. or.). Synonymie générique : Durette-Desset, 1982

Liste des espèces:

belockani (Assadov, 1954) = Ostertagia (Grosspiculagia) belockani Assadov, 1954 = Marshallagia

belockani (Assadov,1954) nov.comb.

brevicauda Hu et Jiang, 1984

brevispiculum Mönnig, 1940

brigantiaca Blanchard in Railliet et Henry, 1909= Ostertagia brigantiaca Blanchard in Railliet et Henry, 1909 / = Ostertagia marshalli Ransom, 1907 fide Gebauer, 1932 = Ostertagia (Marshallagia) marshalli (Ransom,1907) fide Orloff,1933 
dentospicularis Assadov, 1954

marshalli (Ransom, 1907) = Ostertagia marshalli Ransom, 1907 = Ostertagia (Marshallagia) marshalli (Ransom, 1907) Orloff, 1933 = Marshallagia marshalli (Ransom, 1907) Travassos,1937 / = Ostertagia brigantiaca Blanchard in Railliet et Henry, 1909 fide Gebauer,1932, fide Orloff, 1933 / = Ostertagia tricuspis Marotel, 1911 fide Orloff, 1933 /= Ostertagia orientalis Bhalerao,1932 fide Baylis, 1936

mongolica Schumakovitsch, 1938 = Ostertagia (Marshallagia) mongolica Schumakovitsch, 1938 = Marshallagia mongolica (Schumakovitsch, 1938) Skrjabin, Shikhobalova et Schults, 1954

occidentalis (Ransom, 1907) = Ostertagia occidentalis Ransom, 1907 = Ostertagia (Grosspiculagia) occidentalis (Ransom, 1907) Orloff, 1933 = Grosspiculagia occidentalis (Ransom, 1907) Sarwar, 1956 = Ostertagiella occidentalis (Ransom, 1907) Andreeva, 1957a = Marshallagia occidentalis (Ransom, 1907) Durette-Desset, 1982 / = Ostertagia trifida Cuillé, Marotel et Panisset, 1911 fide Gebauer, 1932 / = Ostertagia skrjabini Kamenskii,1929 fide Orloff,1933

orientalis (Bhalerao, 1932) = Ostertagia orientalis Bhalerao, 1932 =Marshallagia orientalis

(Bhalerao, 1932) Travassos, 1937 / = Ostertagia marshalli Ransom, 1907 fide Baylis, 1936 petrovi Assadov, 1959

shikhobalovi Altaev, 1953

sinkiangensis Wu et Shen, 1960

skrjabini Assadov, 1954

tarimanus Qi et $\mathrm{Li}, 1963$

tricuspis Marotel,1911 = Ostertagia tricuspis Marotel, 1911/=Ostertagia (Marshallagia) marshalli (Ransom,1907) fide Orloff,1933/=Ostertagia skrjabini Kamenskii, 1929 fide Skrjabin, Shikhobalova et Schults, 1954

\section{CAMELOSTRONGYLUS Orloff, 1933}

Espèce type : Ostertagia mentula Railliet et Henry, 1909 (dés. or.)

Nous classons dans le genre Camelostrongylus les espèces ayant pour caractères: une bourse caudale de type 2-1-2; des côtes 5 et 6 plus développées que les côtes 2 et 3 et dont les extrémités sont éloignées l'une de l'autre; un synlophe de type Ostertagia.

\section{Synonymes}

Orloffia Drozdz, 1965

Espèce type : Ostertagia orloffi Sankin, 1930 (dés. or.). Synonymie générique: nov.syn. Ostertamia Dikov, 1963

Espèce type : Ostertamia orientalis Dikov, 1963 (mon.). Synonymie générique: nov.syn. 
NOMENCLATURE OSTERTAGIINAE (TRICHOSTRONG YLOIDEA) 359

Sjobergia Sarwar, 1956

Espèce type : Ostertagia lyrata Sjoberg, 1926 (dés. or.). Synonymie générique: Durette- Desset, 1982.

Spiculopteroides Jansen, 1958

Espèce type : Ostertagia odocoilei Dikmans, 1931 (dés. or.). Synonymie générique: nov.syn.

Liste des espèces:

aegagri (Grigoryan,1949) = Ostertagia (Grosspiculagia) aegagri Grigoryan, 1949 = Grosspiculagia aegagri

(Grigoryan, 1949) Jansen, 1958 = Camelostrongylus aegagri (Grigoryan, 1949) nov. comb.

argunica (Rudakov in Skrjabin et Orloff, 1934) = Ostertagia argunica Rudakov in Skrjabin et Orloff, 1934 = Ostertagiella argunica (Rudakov in Skrjabin et Orloff, 1934) Andreeva, 1957a = Came -

lostrongylus argunicus (Rudakov in Skrjabin et Orlof, 1934) nov.comb.

bisonis (Chapin, 1925) = Ostertagia bisonis Chapin, 1925 = Ostertagia (Skrjabinagia) bisonis (Chapin,

1925) Kassimov, 1942 = Skrjabinagia bisonis (Chapin, 1925) Altaev, 1952 = Ostertagiella bisonis

(Chapin,1925) Andreeva, 1957a = Camelostrongylus bisonis (Chapin, 1925) nov.comb.

bondarevi (Andreeva, 1957b) = Skrjabinagia bondarevi Andreeva, 1957b = Camelostrongylus

bondarevi (Andreeva, 1957b) nov.comb.

buriatica (Konstantinova, 1934) = Ostertagia buriatica Konstantinova, 1934 = Ostertagiella buriatica

(Konstantinova, 1934) Andreeva, 1957a = Skrjabinagia buriatica (Konst- tinova, 1934) Andreeva et

Cujko, 1966 = Camelostrongylus buriaticus (Konstantinova, 1934) nov.comb.

butschenevi (Rudakov, 1937) = Ostertagia (Marshallagia) butschenevi Rudakov, 1937 = Skrjabinagia

butschenevi (Rudakov, 1937) Altaev, 1953 = Marshallagia butschenevi (Rudakov, 1937) Skrjabin,

Shikhobalova et Schults, 1954 = Camelostrongylus butschenevi (Rudakov, 1937) nov. comb.

erschovi (Hsü, Ling et Liang, 1957) = Ostertagia erschovi Hsü, Ling et Liang, 1957 = Camelostrongylus erschovi (Hsü, Ling et Liang, 1957) nov.comb. I =Teladorsagia trifurcata (Ransom, 1907) fide Yen, 1963

harrisi (Le Roux, 1930) = Ostertagia harrisi Le Roux, $1930=$ Ostertagiella harrisi (Le Roux, 1930)

Andreeva,1957a = Stadelmannia harrisi (Le Roux, 1930) Sarwar, 1956 = Camelostrongylus harrisi (Le Roux,1930) nov.comb.

hsiungi (Hsü, Ling et Liang, 1957) = Ostertagia hsiungi Hsü, Ling et Liang, 1957 = Came lostrongylus hsiungi (Hsü, Ling et Liang, 1957) nov.comb.

lyratus (Sjoberg, 1926) = Ostertagia lyrata Sjoberg, 1926 = Ostertagia (Grosspiculagia) lyrata (Sjoberg,1926) Neveu-Lemaire, 1936 = Sjobergia lyrata (Sjoberg, 1926) Sarwar, 1956 = Skrjabinagia lyrata (Sjoberg, 1926) Andreeva, 1957b = Grosspiculagia lyrata (Sjoberg, 1926) Jansen, 1958 
= Camelostrongylus lyratus (Sjoberg, 1926) nov. comb. $/$ = Ostertagia occidentalis Ransom, 1907 sensu Gebauer, 1932 fide Travassos, 1937

mentulatus (Railliet et Henry, 1909) = Ostertagia mentulata Railliet et Henry, 1909 = Camelostrongylus mentulatus (Railliet et Henry, 1909) Orloff, 1933 = Marshallagia mentulata (Railliet et Henry, 1909) Durette-Desset, 1982

nemorhaedi (Shults et Kadenatsii, 1950) = Ostertagia (Grosspiculagia) nemorhaedi Shults et Kadenatsii,1950 = Sjobergia nemorhaedi (Shults et Kadenatsii, 1950) Sarwar, 1956 =Skrjabinagia nemorhaedi (Shults et Kadenatsii, 1950) Andreeva,1957b = Grosspiculagia nemorhaedi (Shults et Kadenatsii, 1950) Jansen, $1958=$ Camelostrongylus memorhaedi (Shults et Kadenatsii, 1950) nov. comb.

nianqinqtanggulaensis ( $\mathrm{K}$ 'ung et $\mathrm{Li}, 1965$ ) = Ostertagia nianqinqtanggulaensis $\mathrm{K}$ 'ung et $\mathrm{Li}, 1965$ = Camelostrongylus nianqinqtanggulaensis ( $K^{\prime}$ ung et $L i, 1965$ ) nov.comb.

odocoilei (Dikmans, 1931) = Ostertagia odocoilei Dikmans, 1931= Ostertagia (Skrjabinagia) odocoilei (Dikmans, 1931) Kassimov, 1942 = Skrjabinagia odocoilei (Dikmans, 1931) Altaev, 1952 = Ostertagiella odocoilei (Dikmans,1931) Andreeva,1957a $=$ Spiculopteroides odocoilei (Dikmans,1931) Jansen, 1958 = Apteragia odocoilei (Dikmans,1931) Drozdz,1965 = Mazamastrongylus odocoilei (Dikmans,1931) Jansen,1986 = Camelostrongylus odocoilei (Dikmans, 1931) nov.comb. orientalis $($ Dikov, 1963) $=$ Ostertamia orientalis Dikov, $1963=$ Camelostrongylus orientalis (Dikov, 1963) nov.comb.

orloffi $($ Sankin, 1930) $=$ Ostertagia orloffi Sankin, $1930=$ Ostertagiella orloffi (Sankin, 1930) Andreeva, 1957a = Orloffia orloffi (Sankin, 1930) Drozdz, 1965 = Camelostrongylus orloffi (Sankin, 1930) nov.comb.

ryjikovi (Jancev, 1977) = Skrjabinagia ryjikovi Jancev, 1977 = Camelostrongylus ryjikovi (Jancev,1977) nov.comb.

skrjabini (Kamenskii, 1929) = Ostertagia skrjabini Kamenskii, 1929=Grosspiculagia skrjabini (Kamenskii, 1929) Sarwar, 1956 = Camelostrongylus skrjabini (Kamenskii, 1929) nov. comb. I = Ostertagia (Grosspiculagia) occidentalis (Ransom, 1907) fide Orloff, 1933 / = Ostertagia tricuspis Marotel, 1911 fide Skrjabin, Shikhobalova et Shults, 1954

skrjabini (Shen, Wu et Yen, 1959) nec Kamenskii, 1929 = Ostertagia (Grosspiculagia ) skrjabini Shen, Wu et Yen, 1959

trifida (Cuillé, Marotel et Panisset, 1911) = Ostertagia trifida Cuillé, Marotel et Panisset, 1911 =Grosspiculagia trifida (Cuillé, Marotel et Panisset, 1911) Sarwar,1956 = Ostertagiella trifida (Cuillé, Marotel et Panisset,1911) Andreeva,1957a = Camelostrongylus trifidus (Cuillé, Marotel et Panisset,1911) nov. comb. I = Ostertagia (Grosspiculagia)occidentalis (Cuillé, Marotel et Panisset, 1911) fide Gebauer, 1932 
NOMENCLATURE OSTERTAGIINAE (TRICHOSTRONGYLOIDEA) 361

\section{OSTERTAGIA Ransom, 1907}

Espèce type: Strongylus ostertagi Stiles, 1892 (dés. or.)

Synonymes:

Capreolagic Shults, Andreeva et Kadenatsii in Skrjabin, Shikhobalova et Shults, 1954.

Espèce type : Capreolagia skrjabini Shults et al. in Skrjabin et al., 1954 (mon.). Synonymie générique: Andreeva, 1957a

Gruhneria Sarwar, 1956

Espèce type :Ostertagia gruhneri Skrjabin,1929 (dés. or.). Synonymie générique: Drozdz,1965

Muflonagia Shults, Andreeva et Kadenatsii in Skrjabin, Shikhobalova et Shults, 1954

Espèce type : Muflonagia podjapolskyi Shults et al. in Skrjabin et al., 1954 (mon.). Synonymie générique : Durette-Desset, 1982.

Ostertagiana Dikov, 1963

Espèce type : Ostertagiana andreevi Dikov, 1963 (mon.). Synonymie générique: Durette-Desset, 1982.

Skrjabinagia Kassimov, 1942 (subgen.)

Espèce type : Ostertagia (Skrjabinagia) popovi Kassimov, 1942 (dés. or.) / =Skrjabinagia kolchida

(Popova,1937) fide Drozdz,1965. Elevé au rang générique par Altaev, 1952. Synonymie générique:

Durette-Desset, 1982.

Tunisostertagia Bernard, 1987 (sub. gen.)

Espèce type : Ostertagia (Tunisostertagia) heterospiculum Bernard, 1987 (mon.). Synonymie générique: nov syn.

Liste des espèces :

andreevi (Dikov, 1963) = Ostertagiana andreevi Dikov, 1963 = Ostertagia andreevi (Dikov, 1963)

Durette-Desset, 1982

angusdunni Gibbons et Khalil, 1980

arctica Mitzkewisch, 1929 = Ostertagia (Grosspiculagia) arctica (Mitzkewisch, 1929) Skrjabin,

Shikhobalova et Shults, 1954 = Sjobergia arctica (Mitzkewisch, 1929) Sarwar, 1956 = Ostertagiella

arctica (Mitzkewisch, 1929) Andreeva, 1957a = Grosspiculagia arctica (Mitzkewisch, 1929) Jansen,

1958 = Teladorsagia arctica (Mitzkewisch, 1929) Drozdz, 1965

caprae Andreeva et Nikolsky in Andreeva,1957c / = Ostertagia ostertagi (Stiles, 1892) fide Drozdz, 1965

capreoli Andreeva, 1957c nom. nov. pour Capreolagia skrjabini Shults, Andreeva et Kadenatsii, 1954

capreolagi Jansen, 1958 nom. nov. pour Capreolagia skrjabini Shults, Andreeva et Kadenatsii, 1954

crimensis Kadenatsii et Andreeva, 1958/= Ostertagia leptospicularis Assadov, 1953 fide Drozdz, 1965

dahurica Orloff, Belowa et Gnedina, 1931 = Ostertagiella dahurica (Orloff, Belowa et Gnedina, 1931)

Andreeva, 1957a 
dikmansi Becklund et Walter, 1968 = Skrjabinagia dikmansi (Becklund et Walter, 1968) Drozdz, 1971 drozdzi Jancev, 1977

gruhneri Skrjabin, 1929 = Gruhneria gruhneri (Skrjabin, 1929) Sarwar, 1956 I=Ostertagia (Grosspiculagia ) petrovi Puschmenkov, 1937 fide Drozdz, 1965/ = Ostertagia tatiani Puschmenkov, 1937 fide Drozdz, 1965 / = Ostertagia tundra Gnedina et Antipin in Skrjabin et Orloff, 1934 fide Drozdz, 1965

heterospiculum Bernard, 1987 = Ostertagia (Tunisostertagia) heterospiculum Bernard, 1987

kasakhstanica (Dikov et Nekipelova, 1963) = Teladorsagia kasakhstanica Dikov et Nekipelova, 1963 = Ostertagia kasakhstanica (Dikov et Nekipelova, 1963) nov.comb.

kolchida Popova, 1937 = Sjobergia kolchida (Popova, 1937), Sarwar, 1956= Skrjabinagia kolchida (Popova, 1937) Andreeva, 1957b = Grosspiculagia kolchida (Popova, 1937) Jansen, 1958 I=Ostertagia (Skrjabinagia ) popovi Kassimov, 1942 fide Drozdz, 1965 / = Ostertagia (Grosspiculagia) lasensis Assadov, 1953 fide Drozdz, $1965 /=$ Muflonagia podjapolskyi Shults, Andreeva et Kadenatsii, 1954 fide Drozdz,1965

lasensis Assadov, 1953 =Ostertagia (Grosspiculagia) ) lasensis Assadov, 1953 = Skrjabinagia lasensis (Assadov, 1953) Andreeva,1957b = Grosspiculagia lasensis (Assadov, 1953) Jansen, $1958 /=$ Ostertagia kolchida Popova, 1937 fide Drozdz,1965

leptospicularis Assadov, 1953 / = Capreolagia skrjabini Shults, Andreeva et Kadenatsii,1954 fide Drozdz,1965 = Ostertagia capreoli Andreeva, 1957a = Ostertagia capreolagi Jansen, 1958 / = Capreolagia antipini Kadenatsii, 1957 fide Drozdz, 1965/=Capreolagia paraskrjabini Kadenatsii, 1957 fide Drozdz, 1965 / = Ostertagia paracapreoli Kadenatsii et Andreeva, 1958 fide Drozdz, 1965 I= Ostertagia crimensis Kadenatsii et Andreeva, 1958 fide Drozdz, 1965 / = Ostertagia taurica Kadenatsii et Andreeva, 1958, fide Drozdz, 1965

lyrataeformis $($ Drozdz,1965) = Skrjabinagia lyrataeformis Drozdz, 1965 = Ostertagia lyrataeformis (Drozdz, 1965) nov.comb.

mossi Dikmans, 1931

ostertagi $($ Stiles, 1892 ) = Strongylus ostertagi Stiles, 1892 = Ostertagia ostertagi (Stiles, 1892) Ransom, 1907 / = Ostertagia caprae Andreeva et Nikolsky in Andreeva, 1957c fide Drozdz,1965 paracapreoli Kadenatsii et Andreeva, 1957 / = Ostertagia leptospicularis Assadov, 1953 fide Drozdz, 1965 petrovi Puschmenkov, 1937 = Ostertagia (Grosspiculagia $)$ petrovi Puschmenkov, 1937 = Grosspiculagia petrovi (Puschmenkov, 1937) Jansen, 1958 / = forme tératologique d'Ostertagia gruhneri Skrjabin, 1929 fide Drozdz, 1965 
NOMENCLATURE OSTERTAGIINAE（TRICHOSTRONG YLOIDEA) 363

podjapolsanyi (Shults, Andreeva et Kadenatsii, 1954) = Muflonagia podjapolskyi Shults et al., 1954

= Skrjabinagia podjapolskyi (Shults et al., 1954) Andreeva, 1957b = Grosspiculagia podjapolskyi (Schults et al., 1954) Jansen, 1958 / = Ostertagia kolchida Popova, 1937 fide Drozdz, 1965

polarica Puschmenkov, 1937 = Stadelmannia polarica (Puschmenkov, 1937) Sarwar, 1956 = Ostertagiella

polarica (Puschmenkov,1937) Andreeva, 1957a/ = forme tératologique d'Ostertagia sp. fide Drozdz, 1965

popovi Kassimov, 1942 = Ostertagia (Skrjabinagia ) popovi Kassimov, 1942 = Skrjabinagia popovi (Kassimov, 1942), Altaev, 1952 = Grosspiculagia popovi (Kassimov, 1942), Jansen, $1958 /=O s$ tertagia kolchida Popova, 1937 fide Drozdz, 1965

rubricervi Andrews, 1964 = Ostertagia (Grosspiculagia) rubricervi Andrews, 1964

sissoki Diaouré ,1964

arrjabini (Shults, Andreeva et Kadenatsii, 1954) = Capreolagia skrjabini Shults et al., $1954=$ Ostertagia skrjabini (Schults et al., 1954) Andreeva, 1957c = Ostertagia capreoli Andreeva, 1957c = Ostertagia capreolagi Jansen, 1958 / =Ostertagia leptospicularis Assadov, 1953 fide Drozdz, 1965 tarandi Hellesnes, 1935

tatiani Puschmenkov, 1937 /= forme tératologique d'Ostertagia gruhneri Skrjabin, 1929 fide Drozdz, 1965

taurics Kadenatsii et Andreeva, 1958 / = Ostertagia leptospicularis Assadov, 1953 fide Drozdz,1965

tundra Gnedina et Antipin in Skrjabin et Orloff,1934 = Skrjabinagia tundra (Gnedina et Antipin in Skrjabin et Orloff, 1934) Altaev, 1953 / = forme tératologique d'Ostertagia gruhneri Skrjabin, 1929 fide Drozdz, 1965

volgaensis Tomskich, 1938 = Ostertagia (Grosspiculagia) volgaensis Tomskich, $1938=$ Grosspiculagia volgaensis (Tomskich, 1938) Jansen, 1958

wuhingensis Ling et Chow, 1960

\section{LONGISTRONGYLUS Le Roux, 1931}

Espèce type : Longistrongylus meyeri Le Roux, 1931 (mon.)

Synonymes :

Bigalkea Mönnig, 1931

Espèce type : Bigalkea albifrontis Mönnig, 1931 (mon.). Synonymie générique : Travassos, 1937

Bigalkenema Ortlepp, 1963

Espèce type : Bigalkenema namaquensis Ortlepp, 1963 (dés. or.). Synonymie générique : Gibbons, 1977. 
Costarcuala Shults e: Kadenatsii, 1950 (subgen.)

Espèce type : Ostertagia (Costarcuata) muraschkinzevi Shults et Kadenatsii, 1950 (mon.). Synonymie générique : Jansen, 1958.

Kobusinema Ortlepp, 1963

Espèce type : Longistrongylus schrenki Ortlepp, 1939 (dés. or.). Synonymie générique : Gibbons, 1977.

Pseudomarshallagia Roetti, 1941 (subgen.)

Espèce type : Ostertagia (Pseudomarshallagia) elongata Roetti,1941 (mon.).

Elevé au rang générique par Graber et Delavenay, 1978. Synonymie générique : Durette-Desset, 1982.

Liste des espèces

albifrontis (Mönnig, 1931) = Bigalkea albifrontis Monnig, 1931 = Longistrongylus albifrontis

(Mönnig, 1931) Travassos, 1937

banagiense (Gibbons,1972) = Kobusinema banagiense Gibbons, 1972 = Longistrongylus

banagiense (Gibbons, 1972) Gibbons, 1977

curvispiculum (Gibbons, 1973) = Bigalkenema curvispiculum Gibbons, 1973 =Longistrongylus

curvispiculum (Gibbons,1973) Gibbons, 1977

elongata (Roetti, 1941) = Ostertagia (Pseudomarshallagia elongata) Roetti, 1941 = Pseudomarshallagia

elongate (Roetti, 1941) Graber et Delavenay, 1978 =Longistrongylus elongata (Roetti,

1941) Durette-Desset, 1982

meyeri Le Roux, 1931

muraschkinzevi (Shults et Kadenatsii, 1950) = Ostertagia (Costarcuata) muraschkinzevi Shults et

Kadenatsii, 1950 = Ostertagia muraschkinzevi (Shults et Kadenatsii, 1950) Skrjabin, Shikhobalova et

Shults, 1954 =Longistrongylus muraschkinzevi (Shults et Kadenatsii, 1950)

Jansen, 1958

namaquensis (Ortlepp,1963) = Bigalkenema namaquensis Ortlepp, 1963 = Longistrongylus namaquensis (Ortlepp, 1963) Gibbons, 1977

neveulemairei (Gutteres, 1947) = Ostertagia (Ostertagia) neveulemairei Gutteres, 1947 I = Bigalkea sabie

(Mönnig, 1932) fide Jansen, 1958

sabie (Mönnig,1932) = Bigalkea sabie Mönnig, 1932 =Longistrongylus sabie (Mönnig, 1932)

Travassos, 1937 = Bigalkenema sabie (Mönnig, 1932) Ortlepp, 1963 / =Ostertagia neveulemairei (Gutteres, 1947) fide Jansen, 1958

schrenki Ortlepp, 1939 = Bigalkea schrenki (Ortlepp, 1939) Skrjabin et Shikhobalova in Skrjabin, Shikhobalova, Shults, Popova, Boev et Delamyure, 1952 = Kobusinema schrenki (Ortlepp, 1939) Ortlepp, 1963 
NOMENCLATURE OSTERTAGIINAE (TRICHOSTRONGYLOIDEA) 365

SPICULOPTERAGIA Orloff, 1933 (subgen.)

Espèce type : Osteriagia spiculoptera Gushanskaya (1) ${ }_{\text {in }}$ Skrjabin et Orloff, 1934 (dés de Travassos, 1937)

I= Ostertagia boehmi Gebauer, 1932 fide Orloff, 1933

Elevé au rang générique par Travassos, 1937

Synonymes :

Altaevia Sarwar, 1957

Espèce type : Ostertagia schulzi Raevskaya, 1930 (dés. or.). Synonymie générique : Drozdz,1965.

Apteragia Jansen, 1958

Espèce type : Apteragia quadrispiculata Jansen, 1958 (dés. or.). Synonymie générique: Durette-

Desset, 1982.

Mazamastrongylus Cameron, 1935

Espèce type : Mazamastrongylus trinitatis Cameron, 1935 (mon.). Synonymie générique: Travassos, 1937.

Petrowagia Ruchliadev, 1961 (subgen.)

Esp.-type : Spiculopteragia (Petrowagia) pigulevski Ruchliadev, 1961 (mon.). Synonymie générique: Hinaidy, Gutierres et Supperer, 1972.

Rinadia Grigoryan, 1951

Esp.-type : Rinadia sch:llzi Grigoryan, 1951 (mon.) /=Rinadia mathevossiani (Ruchliadev,1948)

fide Drozdz,1965. Synonymie générique: Durette-Desset,1982.

Sarwaria Drozdz, 1965

Esp.-type : Gruhneria bubalis Sarwar, 1956 (mon.). Synonymie générique: Durette-Desset,1982.

Liste des espèces

alcis Shults, Kadenatsii, Evranova et Schaldybin, in Skrjabin, Shikhobalova, Shults, Popova, Boev et Delyamure, 1952 = Altaevia alcis (Shults et al., in Skrjabin et al, 1952) Sarwar, $1957=$ Mazamastrongylus alcis (Shults et al., in Skrjabin et al., 1952) Jansen, $1958 /=$ Spiculopteragia dagestanica (Altaev, 1953) fide Drozdz, 1965

asymmetrica (Ware, 1925) = Ostertagia asymmetrica Ware, $1925=$ Ostertagia (Spiculopteragia) asymmetrica (Ware,1925) Orloff, 1933 = Spiculopteragia asymmetrica (Ware, 1925) Travassos, 1937 / = Spiculopteragia cervi (Cameron, 1931) fide Hinaidy et Prosl, 1981

(1) Le nom existe en manuscrit en1931; il n'est pas valable à cette date et, si la synonymie se révélait être exacte, le taxon $S$. boehmi deviendrait l'espèce-type. 
bоейmi (Gebauer, 1932) = Ostertagia boekmi Gebauer, 1932 =Spiculopteragia boehmi (Gebauer,1932) Skrjabin, Shikhobalova et Shults,1954 / = Ostertagia (Spiculopteragia) spiculoptera (Gushanskaya,1931 ms.) fide Orloff, 1933, fide Skrjabin et Orloff, 1934, fide Travassos,1937 / = Spiculopteragia kutkascheni Assadov, 1952 fide Hinaidy, Gutierres et Supperer, 1972 / = Spiculopteragia (Petrowagia) pigulevski Ruchliadev, 1961 fide Hinaidy, Gutierres et Supperer, 1972

boevi (Pande et Chauhan, 1969) = Skrjabinagia boevi Pande et Chauhan, $1969=$ Spiculopteragia boevi (Pande et Chauhan, 1969) nov.comb. I = Sarwaria bubalis (Sarwar, 1956) fide Hinaidy et Prosl, 1981

bubalis $($ Sarwar, 1956) $=$ Gruhneria bubalis Sarwar, $1956=$ Sarwaria bubalis (Sarwar, 1956) Drozdz, 1965 = Spiculopteragia bubalis (Sarwar, 1956) Durette-Desset, 1982 = Mazamastrongylus bubalis (Sarwar, 1956) Jansen, 1986 I =Skrjabinagia boevi Pande et Chauhan, 1969 fide Hinaidy et Prosl, 1981

caballeroi Chabaud, 1976

caucasica (Assadov, 1955) = Rinadia caucasica Assadov, 1955 = Spiculopteragia caucasica (Assadov, 1955) nov. comb. / = Rinadia mathevossiani Ruchliadev, 1948 fide Drozdz, 1965

cervi (Cameron, 1931) = Ostertagia cervi Cameron, 1931 = Ostertagia (Spiculopteragia) cervi (Cameron, 1931) Orloff, 1933 = Spiculopteragia cervi (Cameron, 1931) Travassos, 1937 = Skrjabinagia cervi (Cameron, 1931) Altaev, 1952/ = Spiculopteragia asymmetrica (Ware, 1925) fide Hinaidy et Prosl, 1981

dagestanica (Altaev, 1953) = Skrjabinagia dagestanica Altaev, 1953 = Altaevia dagestanica (Altaev, 1953) Sarwar, 1957 = Spiculopteragia dagestanica (Altaev,1953) Andreeva, 1957a = Spiculopteroides dagestanica (Altaev, 1953) Jansen, 1958 = Mazamastrongylus dagestanica (Altaev, 1953) Jansen, 1986 / = Spiculopteragia alcis Shults et al. in Skrjabin et al. 1952 fide Drozdz, 1965 I = Spiculopteragia popovi Evranova, 1954 fide Drozdz, 1965

houdemeri (Schwartz, 1926) = Ostertagia houdemeri Schwartz, 1926 = Ostertagia (Spiculopteragia) houdemeri (Schwartz,1926) Orloff,1933 = Spiculopteragia houdemeri (Schwartz, 1926) Travassos, 1937 = Skrjabinagia houdemeri (Schwartz,1926) Altaev,1952 / =Spiculopteragia yamashitai Ohbayashi,1961 fide Hinaidy, Gutierres et Supperer, 1972

janseni $($ Drozdz, 1965) = Apteragia janseni Drozdz,1965 = Mazamastrongylus janseni (Drozdz, 1965) Jansen,1986 = Spiculopteragia janseni (Drozdz, 1965) nov.comb.

kutkascheni Assadov, 1952/ =Spiculopteragia spiculoptera (Gushanskaya, in Skrjabin et Orloff, 1934) fide Jansen, 1958 / = Spiculopteragia boehmi (Gebauer, 1932) fide Hinaidy, Gutierres et Supperer, 1972 
NOMENCLATURE OSTERTAGIINAE (TRICHOSTRONGYLOIDEA) 367

mathevossiani Ruchliadev, 1948 = Rinadia mathevossiani (Ruchliadev, 1948) Andreeva, 1957c / = Rinadia schulzi Grigoryan, 1951 fide Jansen, 1958 / = Rinadia caucasica Assadov, 1955 fide Drozdz,1965 I = Rinadia pavlovskyi Kadenatsii et Andreeva, 1957 fide Drozdz, 1965 / = Rinadia quadrifurcata Andrews, 1964 fide Hinaidy et Prosl, 1981

pavlovskyi (Kadenatsii et Andreeva, in Andreeva, 1957c) = Rinadia pavlovskyi Kadenatsii et Andreeva, in Andreeva, 1957c = Spiculopteragia pavlovskyi (Kadenatsil et Andreeva, in Andreeva, 1957c) nov. comb. $/$ = Rinadia mathevossiani (Ruchliadev, 1948) fide Drozdz, 1965

pigulevski Ruchliadev, 1961 = Spiculopteragia (Petrowagia) pigulevski Ruchliadev, 1961 / =Spiculopteragia spiculoptera (Guschanskaya, in Skrjabin et Orloff, 1934) fide Drozdz, 1965 / = Spiculopteragia boehmi (Gebauer, 1932) fide Hinaidy, Gutierres et Supperer, 1972

popovi Evranova, 1954 / = Spiculopteragia alcis Schults et al. in Skrjabin et al., 1952 fide Andreeva, 1957a / = Spiculopteragia dagestanica (Altaev, 1953) fide Drozdz, 1965

quadrifurcata Andrews, 1964 / = Rinadia mathevossiani (Ruchliadev, 1961) fide Hinaidy et Prosl, 1981 quadrispiculata (Jansen, 1958) = Apteragia quadrispiculata Jansen, 1958 =Spiculopteragia quadrispiculata (Jansen, 1958) Durette-Desset, $1982 /=S k r j a b i n a g i a$ monodigitata Andrews, 1964 fide Hinaidy et Prosl, 1981

schulzi (Raevskaya, 1930) = Ostertagia schulzi Raevskaya, 1930 = Ostertagia (Spiculopteragia) schulzi (Raevskaya, 1930) Orloff, 1933 = Spiculopteragia schulzi (Raevskaya, 1930) Travassos, 1937 = Altaevia schulzi (Raevskaya , 1930) Sarwar, 1957 schulzi (Grigoryan, 1951) nec Raevskaya, 1930 = Rinadia schulzi Grigoryan, $1951=$ Spiculopteragia schulzi (Grigoryan, 1951) Durette-Desset, $1982 /$ = Rinadia mathevossiani (Ruchliadev, 1948) fide Jansen, 1958

spiculoptera (Gushanskaya in Skrjabin et Orloff, 1934) = Ostertagia (Spiculopteragia) spiculoptera (Gushanskaya, 1931 ms.) Orloff, 1933 = Ostertagia (Spiculopteragia) spiculoptera Gushanskaya, 1931 ms. in Skrjabin et Orloff, 1934 =Spiculopteragia spiculoptera (Gushanskaya in Skrjabin et Orloff,1934) Travassos, $1937 /$ = Ostertagia boehmi Gebauer, 1932 fide Skrjabin et Orloff, 1934 / = Spiculopteragia kutkascheni Assadov, 1952 fide Jansen, $1958 /=S p i$ culopteragia (Petrowagia) pigulevski Ruchliadev, 1961 fide Drozdz, 1965

suppereri Hinaidy et Prosl, 1978.

trinitatis (Cameron, 1935) = Mazamastrongylus trinitatis Cameron, $1935=$ Spiculopteragia trinitatis (Cameron, 1935) Travassos, 1937

yamashitai Ohbayashi, 1966 = Spiculopteragia houdemeri (Schwartz, 1926) fide Hinaidy, Gutierres et Supperer, 1972 


\section{TELADORSAGIA Andreeva et Satubaldin,1954}

Espèce type : Teladorsagia davtiani sensu Andreeva et Satubaldin in Skrjabin, Shikhobalova et Shults, 1954 nec Grigoryan, 1951 (mon.)

I = Teladorsagia trifurcata (Ransom, 1907) fide Drozdz, 1965

\section{Synonymes:}

Dorsagia Guerrero et Chavez, 1967 (subgen.)

Espèce type : Spiculopteragia (Dorsagia) peruvianus Guerrero et Chavez, 1967 (mon.). Synonymie générique: Durette-Desset, nov. syn.

Paramecistocirrus Roetti, 1941

Espèce type : Paramecistocirrus dimorphus Roetti, 1941 (mon.). Synonymie générique : DuretteDesset, 1983.

Stadelmannia Sarwar, 1956.

Espèce type : Ostertagia trifurcata Ransom, 1907 (dés. or.). Synonymie générique : Drozdz, 1965

Liste des espèces :

andreevae (Drozdz, 1965) = Rinadia andreevae Drozdz, 1965 = Teladorsagia andreevae (Drozdz,1965) nov.comb. I =Rinadia japonica Ohbayashi, 1966 fide Hinaidy et Prosl,1981

bakuriani (Schischkin, 1937) = Ostertagia bakuriani Schischkin, 1937 = Ostertagiella bakuriani (Schischkin, 1937) Andreeva, 1957a = Stadelmannia bakuriani (Schischkin, 1937) Sarwar, 1956 = Teladorsagia bakuriani (Schischkin, 1937) nov.comb.

circumcincta (Stadelmann, 1894) = Strongylus circumcinctus Stadelmann, 1894 = Ostertagia circumcincta (Stadelmann, 1894) Ransom, 1907= Stadelmannia circumcincta (Stadelmann,1894) Sarwar, 1956 = Ostertagiella circumcincta (Stadelmann, 1894) Andreeva , 1957a = Teladorsagia circumcincta (Stadelmann, 1894) Drozdz, $1965 /$ = Ostertagia turkestanica Petrov et Shakhovtseva, 1926 fide Orloff, 1933

dactylospicula Wu, Yen et Shen, 1965 = Skrjabinagia dactylospicula Wu, Yen et Shen, 1965 = Teladorsagia dactylospicula (Wu, Yen et Shen, 1965) nov. comb.

davtiani (Grigoryan, 1951) = Ostertagia davtiani Grigoryan, 1951 = Ostertagiella davtiani (Grigoryan, 1951), Andreeva, 1957a = Teladorsagia grigoriani Drozdz, 1965

davtiani Andreeva et Satubaldin in Skrjabin, Shikhobalova et Shults, 1954 nec Grigoryan, 1951 / = Teladorsagia trifurcata (Ransom, 1907) Drozdz, 1965/ = Stadelmannia trifurcata (Ransom, 1907) fide Drozdz, 1965

dimorphus $($ Roetti, 1941) = Paramecistocirrus dimorphus Roetti, 1941 = Teladorsagia dimorpha (Roetti, 1941) nov.comb. 
grigoriani Drozdz, 1965 nom. nov. pour Ostertagia davtiani sensu Grigoryan, 1951 nec Andreeva et Satubaldin, 1954

hamata (Mönnig, 1932) = Ostertagia hamata Monnig, 1932 = Spiculopteragia hamata (Mönnig, 1932)

Travassos, 1937 = Apteragia hamata (Mönnig, 1932) Jansen, 1958 = Teladorsagia hamata (Monnig, 1932) nov.comb.

japonica (Ohbayashi, 1966) = Rinadia japonica Ohbayashi, 1966 = Teladorsagia japonica

(Ohbayashi, 1966) nov.comb. I= Rinadia andreevae Drozdz, 1965 fide Hinaidy et Prosl, 1981 kegeni (Satubaldin in Andreeva, 1957c) = Ostertagiella kegeni (Satubaldin in Andreeva, 1957c)

= Teladorsagia kegeni (Satubaldin in Andreeva, 1957c) Drozdz, 1965

kenyensis (Gibbons et Khalil, 1980) = Ostertagia. kenyensis Gibbons et Khalil, $1980=$ Teladorsagia kenyensis (Gibbons et Khalil, 1980) nov. comb.

monodigitata (Andrews, 1964 ) = Skrjabinagia monodigitata Andrews, 1964 / = Apteragia quadrispiculata Jansen, 1958 fide Hinaidy et Prosl, 1981

panticola $($ Shaldibin, 1964) $=$ Spiculopteragia panticola Shaldibin, $1964=$ Teladorsagia panticola (Shaldibin, 1964) nov.comb.

peruvianus (Guerrero et Chavez, 1967) = Spiculopteragia (Dorsagia) peruvianus Guerrero et Chavez, 1967 = Spiculopteroides peruvianus (Guerrero et Chavez, 1967) Hinaidy et Prosl, 1981 = Teladorsagia peruviana (Guerrero et Chavez, 1967) nov.comb.

pinnata (Daubney, 1933) = Ostertagia pinnata Daubney, 1933 = Ostertagia (Ostertagia) pinnata (Daubney, 1933) Neveu-Lemaire, 1936 = Stadelmannia pinnata (Daubney, 1933) Sarwar, 1956 = Teladorsagia pinnata (Daubney, 1933) Drozdz, $1965 /$ = Ostertagia trifurcata Ransom, 1907 fide Travassos, 1937

pursglovei (Davidson et Prestwood, 1979) = Apteragia pursglovei Davidson et Prestwood, $1979=$ Spiculopteroides pursglovei (Davidson et Prestwocd, 1979) Hinaidy et Prosl, 1981 = Mazamastrongylus pursglovei (Davidson et Prestwood, 1979) Jansen, 1986 = Teladorsagia pursglovei (Davidson et Prestwood, 1979) nov. comb.

trifurcata (Ransom, 1907) = Ostertagia trifurcata Ransom, 1907 = Stadelmannia trifurcala (Ransom, 1907) Sarwar,1956 = Ostertagiella trifurcata (Ransom, 1907) Andreeva, 1957a =Teladorsagia trifurcata (Ransom,1907) Drozdz, $1965 /$ = Ostertagia pinnata Daubney, 1933 fide Travassos, 1937 I = Teladorsagia davtiani Andreeva et Satubaldin, 1954 nec Grigoryan, 1951 fide Drozdz, 1965 I = Ostertagia erschovi Hsu, Ling et Liang, 1957 fide Yen, 1963

turkestanica (Petrov et Shakhovtseva, 1926) = Ostertagia turkestanica Petrov et Shakhovtseva, 1926 = Stadelmannia turkestanica (Petrov et Shakhovtseva,1926) Sarwar, 1956 = Teladorsagia turkestanica (Petrov et Shakhovtseva,1926) Drozdz, 1965/=Ostertagia (Ostertagia) circumcincta (Stadelmann, 1894) fide Orloff, 1933 
vietnamica $($ Drodz, 1965) = Spiculopteragia vietnamica Drozdz, 1965 = Spiculopteroides vietnamica (Drozdz, 1965) Hinaidy et Prosl, 1981 = Mazamastrongylus vietnamica (Drozdz, 1965) Jansen, 1986 = Teladorsagia vietnamica (Drozdz, 1965) nov.comb.

\section{Espèces non classées}

Il s'agit d'espèces dont nous n'avons pas pu obtenir la description originale ou dont la description est insuffisante pour permettre un classement.

antipini Kadenatsii, 1957 (Capreolagia) / = Ostertagia leptospicularis Assadov, 1953 fide Drozdz, 1965 murmani Tachistow, 1940 (Ostertagia)

paraskrjabini Kadenatsii, 1957 (Capreolagia) / = Ostertagia leptospicularis Assadov, 1953 fide Drozdz, 1965

schumakovitshi Kadyrov, 1959 (Ostertagia)

\section{Species inquirendae:}

Ostertagia antipini Matschulskii, 1950

Trichostrongyella schulzi Dikov, 1961

\section{Espèces exclues des Ostertagiinae}

Ostertagia (Grosspiculagia) skrjabini sensu Singh et Pande, $1963=$ [Ostertagia (Grosspiculagia) malviyai Chatuverdi et Kansal, 1977], espèce-type du genre Cervicaprastrongylus Gibbons et Khalil, 1982, doit à notre avis être classé dans les Graphidiinae, ce genre étant proche ou synonyme d'Hyostrongylus Hall, 1921.

Ostertagia thalae Troncy et Graber, 1973 doit à notre avis être rangé dans les Graphidiinae.

Bergheia okapiae (Berghe, 1937) Drozdz, 1965 = Hyostrongylus okapiae (Berghe, 1937) Jansen, 1958, a été classé dans les Graphidiinae par Durette-Desset, 1983.

Gazellostrongylus lerouxi Yeh, 1956 a été classé dans les Cooperiinae par Durette-Desset, 1983.

Remerciement. - Nous remercions Monsieur le Professeur Claude Dupuis de ses précieux conseils concernant la nomenclature.

\section{RÉFÉRENCES}

Une liste exhaustive des travaux traitant des Ostertagiinae constituerait un véritable volume. Nous ne donnons donc ici que les références des travaux qui ont traité de la systématique générale de ce groupe.

Andreeva N. K. : Revizia Ostertagiei (Trichostrongylidy) zvacnych. Trudy Inst. Vet. Kazfiliala, 1957a, 8, 473-487. 
Andreeva N. K. : Revizia Skrjabinagiei (Trichostrongylidy) zvacnych. Trudy Inst. Vet. Kazfiliala, $1957 \mathrm{~b}, 8,488-497$.

Andreeva N. K. : Atlas des Helminthes (Strongylata) de ruminants domestiques et sauvages de Kazakstan. Tashkent, Kazakstan, $1957 \mathrm{c}, 215 \mathrm{p}$.

Baylis H. A. : The Fauna of British India, including Ceylon and Burma. Nematoda. Vol. I. Ascaroidea and Strongyloidea. Taylor and Francis, 1936, London, xxxvi $+408 \mathrm{p}$.

Drozdz J. : Studies on helminths and helminthiases in Cervidae. I. Revision of the subfamily Ostertagiinae Sarwar, 1956 and an attempt to explain the phylogenesis of its representatives. Acta Parasit. Pol., 1965, 13, 445-481.

Durette-Desset M.-C. : Sur les divisions génériques des Nématodes Ostertagiinae (Trichostrongylidae). Annls Parasitol. Hum. Comp., 1982, 57, 375-381.

Durette-Desset M.-C. : Keys to genera of Superfamily Trichostrongyloidea. In CIH Keys to the Nematode Parasites of Vertebrates. R. S. Anderson \& Chabaud A. G. Ed. Commonwealth Agricultural Bureaux, Fairham Royal, Bucks, England, 1983, 1-68.

Ferté H. : Les Nématodes parasites du tube digestif du Chevreuil (Capreolus capreolus L., 1758). A propos d'une enquête réalisée dans le Nord-Est de la France. Thèse de Doctorat de troisième cycle dans les disciplines pharmaceutiques. Académie d'Orléans-Tours, 1987, 165 p., polycopié.

Gibbons L. M. : Revision of the genera Longistrongylus Le Roux, 1931, Kobusinema Ortlepp, 1963 and Bigalkenema Ortlepp, 1963 (Nematoda: Trichostrongylidae). J. Helminth., 1977, $51,41-62$.

Gibbons L. M., Khalil L. F. : A key for the identification of genera of the nematode family Trichostrongylidae Leiper, 1912. J. Helminth., 1982, 56, 185-233.

Hinaidy H. K., Prosl H. : Proposals to the systematics of Ostertagia species: tribe spiculopteragiini. In Third European Multicolloquium of Parasitology, Cambridge, 1980, September 7-13, Workshop proceed, $\mathrm{n}^{\circ} 14$. Parasitology, 1981, 82, 181-183.

JANSEn J. : Lebmaagtrichostrongyliden bij Nederlanse Herten. Drukkeri H. J. Smits Ed., Utrecht, 1958, 100 p. (Résumé anglais).

JANSEN J. : Spiculopteragia (Orloff, 1933) and related genera. In Third European Multicolloquium of Parasitology, Cambridge, 1980, September 7-13, Workshop proceed, no 14. Parasitology, 1981, 82, 183-185.

Jansen J., Gibbons L. M. : Systematics and biology of Ostertagia sensu lato (Nematoda: Trichostrongylidae). In Third European Multicolloquium of Parasitology, Cambridge, 1980, September 7-13, Workshop proceed, no 14. Parasitology, 1981, 82, 175-189.

Khalil L. F., Gibbons L. M. : The subfamilly Ostertagiinae Sarwar, 1956. In Third European Multicolloquium of Parasitology, Cambridge, 1980, September 7-13, Workshop proceed, no 14. Parasitology, 1981, 82, 177-181.

Sarwar M. M. : Studies on some trichostrongylids of ruminants from the Indo-Pakistan Subcontinent. Biologia, Lahore, 1956, 2, 146-215.

Skrjabin K. I., Orlov I. V. : Trichostrongylose des Ruminants. Bibliothèque d'Helminthologie (Pr K. I. Skrjabin), Moscou Leningrad, 1934, 351 p.

Skrjabin K. I., Shikhobalova N. P., Shults R. S. : Osnovy nematodolgii. IV. Diktyokaulidy, Geligmozomatidy i Ollulanidy zhivotnykh. Acad. Sciences U. R. S. S., Moscou, 1954, 323 p.

Travassos L. : Revisao da familia Trichostrongylidae Leiper, 1912. Monogr. Inst. Oswaldo Cruz, 1937, I, $512 \mathrm{p}$.

Pages 372 et 373 : Index des noms de genres et d'espèces. 
INDEX DES NOMS DE GENRES

\begin{tabular}{|c|c|c|c|c|c|}
\hline Allaevia & Sarwar, 1957 & 365 & Astertagia & Ransom, 1907 & 361 \\
\hline Apteragio & Jansen, 1958 & 365 & istertagiana & Dikar, 1963 & 361 \\
\hline Bergheia & Drozdz, 1965 & 370 & Oster tagiella & Andreeva, 1956 & 357 \\
\hline Bigalkea & Mönnig, 1931 & 363 & Ostertamia & Dikov, 1963 & 358 \\
\hline Bigalkenema & Ortlepp, 1963 & 363 & Paramecistacirrus & Roetti, 1941 & 368 \\
\hline Camelostrongylus & Orloff, 1933 & 358 & Petrowiaga & Ruchljadev, 1961 & 365 \\
\hline Caprealogia & Shults,et al., 1953 & 361 & Pseudomarshallagl & Roetti, 1941 & 364 \\
\hline Costarcuata & Shults et al. 1950 & 364 & Rinadia & Grigoryan, 1951 & 365 \\
\hline Qazellastrongylus & Yeh, 1956 & 370 & Sarwaria & Drozdz, 1965 & 365 \\
\hline Dorsagia & Guerrero et al. , 1967 & 368 & Siohergia & Sarwar, 1956 & 359 \\
\hline Grosspiculagia & Or loff, 1933 & 357 & Skriabinagia & Kassimov, 1942 & 361 \\
\hline Gruhneria & Sarwar, 1956 & 361 & spicuiapteragia & Orloff, 1933 & 365 \\
\hline Kobusinema & Ortlepp, 1963 & 364 & Spiculopter orides & Jansen, 1958 & 359 \\
\hline Langistrongylus & Le Roux, 1931 & 363 & Staueimannia & Sarwar, 1956 & 368 \\
\hline Marshallagia & Orloff, 1933 & 357 & Teladorsagia & Andreeva et al. , 1954 & 368 \\
\hline Mazamastrongylus & Cameron, 1935 & 365 & Trianastrangualla & Dikov, 1961 & 370 \\
\hline Muflonagia & Shults et al. , 1954 & 361 & Tunisusterabia & Bernard, 1987 & 361 \\
\hline Orloffia & Drozdz, 1965 & 358 & & & \\
\hline
\end{tabular}

\section{INDEX DES NOMS D'ESPECES}

\begin{tabular}{|c|c|c|c|c|c|}
\hline Degagri & Grigoryan, 1949 & 359 & Capranlian & Jansen, 1958 & 361 \\
\hline albifrantis & Mönnig, 1931 & 364 & oucusina & Assadov, 1955 & 366 \\
\hline alcis & Shults et al., 1954 & 365 & carvi & Cameron, 1931 & 366 \\
\hline andreevae & Drozdz, 1965 & 368 & circumcincta & Stadelmann, 1894 & 368 \\
\hline andreevi & Dikov, 1963 & 361 & crimensis & Kadenatsii et al. , 1958 & 361 \\
\hline angusdunni & Gibbons et al. , 1980 & 361 & curvispiculum & Gibbons, 1973 & 364 \\
\hline antipini & Matschulskii, 1.950 & 370 & dartylospicula & Shu-Wing et al. 1965 & 368 \\
\hline antipini & Kadenatsii, 1957 & 370 & dagestanica & Altaev, 1952 & 366 \\
\hline arctica & Mitzkewisch, 1929 & 361 & dahurica & Orloff et al. , 1931 & 361 \\
\hline argunica & Rudakov, 1934 & 359 & davtiani & Andreeva et al. , 1953 & 368 \\
\hline asymmetrica & Ware, 1925 & 365 & davtiani & Grigoryan, 1951 & 368 \\
\hline bakuriani & Schischkin, 1937 & 368 & dentospicularis & Assadov, 1954 & 358 \\
\hline banagiense & Gibbons, 1972 & 364 & dikmansi & Becklund et al, 1968 & 362 \\
\hline belockani & Assadoy, 1954 & 357 & dimorphus & Roetti, 1941 & 368 \\
\hline bisonis & Chapin, 1925 & 359 & drazuti & Jancev, 1981 & 362 \\
\hline boehmi & Gebauer, 1932 & 366 & elangata & Roetti, 1941 & 364 \\
\hline baevi & Pande et al., 1969 & 366 & ersochovi & Hsü et al., 1957 & 359 \\
\hline bandarevi & Andreeva, 1956 & 359 & grigoriani & Drozdz, 1965 & 369 \\
\hline brevicauda & Hu et Jiang, 1984 & 357 & gruhneri & Skrjabin, 1929 & 362 \\
\hline brevispiculum & Mönnig, 1940 & 357 & hamata & Mönnig, 1932 & 369 \\
\hline brigantiaca & Blanchard, 1909 & 357 & harrisi & Le Roux, 1930 & 359 \\
\hline bubalis & Sarvar, 1956 & 366 & heterospiculum & Bernard, 1987 & 362 \\
\hline buriatica & Konstantinova, 1934 & 359 & houdemeri & Schwartz, 1927 & 366 \\
\hline butschnevi & Rudakov, 1937 & 359 & hsiungi & Hsü et al., 1957 & 359 \\
\hline caballerai & Chabaud, 1976 & $366 \cdot$ & janseni & Drozdz, 1965 & 366 \\
\hline caprae & Andreeva et al. , 1957 & 361 & japonioa & Onbayashi, 1966 & 369 \\
\hline capreali & Andreeva, 1957 & 361 & kasaknstaniza & Dikov et al. , 1963 & 362 \\
\hline
\end{tabular}


INDEX DES NOMS D'ESPECES

\begin{tabular}{|c|c|c|c|c|c|}
\hline kegeni & Shatubaldin 1957 & 369 & polarica & Pushmenkov, 1937 & 363 \\
\hline kenyensis & Gibbons et al. 1980 & 369 & popori & Kassimor, 1942 & 363 \\
\hline kolchida & Popova, 1937 & 362 & popovi & Evranova, 1954 & 367 \\
\hline kutkascheni & Assadov, 1952 & 366 & pursglovej & Davidson et al. , 1979 & 369 \\
\hline lasensis & Assadov, 1953 & 362 & quadrifurcata & Andrews, 1964 & 367 \\
\hline leptospicular is & Assadov, 1953 & 362 & auadrispiculata & Jansen, 1958 & 367 \\
\hline lerauxi & Yeh, 1956 & 370 & rubricervi & Andrews, 1964 & 363 \\
\hline Iyratus & Sjoberg, 1926 & 359 & ryikovi & Jancev, 1981 & 360 \\
\hline yrataeformis & Dorzdz, 1965 & 362 & sabie & Mönnig, 1932 & 364 \\
\hline marshalli & Ransom, 1907 & 358 & sochrenki & Ortlepp, 1939 & 364 \\
\hline mathevossiani & Ruchliadev, 1948 & 367 & schulai & Dikov, 1961 & 370 \\
\hline mentulatus & Rallilet et al., 1909 & 360 & sonulej & Raevskaya, 1930 & 367 \\
\hline meyeri & Le Roux, 1931 & 364 & sohulei & Grigoryan, 1951 & 367 \\
\hline mongalica & Schumakovitsch, 1938 & 358 & schumakovitshi & Kadyrov, 1959 & 370 \\
\hline monodigitata & Andrews, 1964 & 369 & shikhobalovi & Altaev, 1953 & 358 \\
\hline mossi & Dikmans, 1931 & 362 & sinkiangensis & Wu et Shen, 1960 & 358 \\
\hline muraschkinzevi & Shults et al. 1950 & 364 & sissoki & Diaouré, 1964 & 363 \\
\hline murmani & Tachistow, 1940 & 370 & skriabini & Kamenstkii, 1929 & 360 \\
\hline namaquensis & Ortlepp, 1963 & 364 & skrjabini & Assadov, 1954 & 358 \\
\hline nemorhaedi & Shults et al. 1950 & 360 & skrianini & Shen et al., 1959 & 360 \\
\hline neveulemairi & Gutteres, 1947 & 364 & skrjabini & Shults et al. 1954 & 363 \\
\hline nianquinq... & K'ung et Li,1965 & 360 & skrjabini & Singh et Pande, 1963 & 370 \\
\hline accidentalis & Ransom, 1907 & 358 & spiculopters & Guschanskaja, 1931 & 367 \\
\hline ocococilei & Dikmans, 1931 & 360 & suppereri & Hinaidy et Prosl, 1976 & 367 \\
\hline okapiae & Berghe, 1937 & 370 & tarandi & Hellesnes, 1935 & 363 \\
\hline orientalis & Bhalerao, 1932 & 358 & tarimanus & Qi et Li, 1963 & 358 \\
\hline orientalis & Dikov, 1963 & 360 & tatiani & Pushmenkov, 1937 & 363 \\
\hline orlorfi & Sankin, 1930 & 360 & tauring & Kadenatsil et al. 1956 & 363 \\
\hline ostertagi & Stiles, 1892 & 362 & thalae? & Trancy et Graber 1973 & 370 \\
\hline panticala & Shaldibin, 1964 & 369 & triauspis & Marotel, 1912 & 358 \\
\hline paracapreoli & Kadenatsii et al., 1957 & 362 & trificte & Guillé et al. , 1911 & 360 \\
\hline paraskrjabini & Kadenatsii, 1957 & 370 & trifurcata & Ransom, 1907 & 369 \\
\hline pavlovskyi & Kadenatsii et al. , 1957 & 367 & trinitatis & Cameron, 1935 & 367 \\
\hline peruvianus & Guerrero et al., 1967 & 369 & tundra & Gnedina et al. , 1934 & 363 \\
\hline petrovi & Puschmenkov, 1937 & 362 & turkestanica & Petrov et al., 1926 & 369 \\
\hline petrovi & Assadov, 1959 & 358 & vietnamica & Drozdz, 1965 & 370 \\
\hline pigulersk: & Ruchliadev, 1961 & 367 & volgensis & Tumskich, 1938 & 363 \\
\hline pinnata & Daubney, 1933 & 369 & whingensis & Ling et Chow, 1960 & 363 \\
\hline podpiopolsky & Shults et al., 1954 & 363 & yamashitai & Ohbayashi, 1966 & 367 \\
\hline
\end{tabular}

